\title{
Selección natural y construcción de nicho: una ¿dialéctica? evolucionista
}

\section{Natural selection and niche construction: An evolu- tionary «dialectic»}

\author{
JUAN RAMÓN ÁLVAREZ \\ Universidad de León (España)
}

Recibido: 7-1-2013

Aprobado definitivamente: 7-2-2013

\section{RESUMEN}

En el marco del pensamiento evolucionista de los últimos treinta años, la teoría de construcción de nicho ha ido abriéndose paso como una perspectiva opuesta a y complementaria de la teoría de la selección natural en la explicación del proceso evolutivo. El planteamiento que sigue aborda su oposición como un proceso de combinación de principios ecológicos (restrictivos) y técnicos (transformadores) que tienden un puente entre ciencias biológicas y ciencias humanas, basado en una analogía de la técnica que se naturaliza en procesos de trasformación en que los organismos «se trabajan» sus ambientes.

$$
\text { PALABRAS CLAVE }
$$

CONSTRUCCIÓN DE NICHO, DIALÉCTICA, ECOLOGÍA, TÉCNICA, SELECCIÓN NATURAL

\footnotetext{
ABSTRACT

Within the frame of evolutionary thought during the last thirty years, niche construction theory has been gaining ground as an opposed and complementary outlook regarding natural selection theory in the explanation of evolution. The following approach construes their opposition as a combination of ecologic (restrictive) and technologic (transformational) principles that serve as 
a bridge between biological and human sciences, based on an analogy with technology that is naturalized in terms of transformation processes wherein organisms «do their work on» their environments.

KEYWORDS

DIALECTICS, ECOLOGY, NATURAL SELECTION, NICHE CONSTRUCTION, TECHNOLOGY

Human beings, like all other organisms, are «active subjects transforming nature according to its laws» and are always in the course of adapting to the ecosystems they themselves construct. (D. Harvey, 1993, p. 28)

\section{INTRODUCCIÓN}

RECIENTEMENTE SE ESTÁN PRODUCIENDO INTENTOS de convergencia para unificar bajo planteamientos unitarios las ciencias biológicas y las ciencias humanas. Dos intentos parecen singularmente interesantes. Son, por una parte, la introducción de la teoría de la construcción de nicho entrelazada y complementando la teoría de la selección natural en el programa evolucionista y, por otra, la proyección de la teoría de juegos como red unificadora de las ciencias del comportamiento. Al primero dan credibilidad trabajos como los de John Odling-Smee, Kevin Laland y Marcus Feldman; al segundo sobre todo la iniciativa encabezada por Herbert Gintis. El grueso de estos planteamientos, aun cuando han tenido antecedentes importantes con anterioridad, se ha desarrollado a partir de la última década del siglo pasado y la que va de éste. En ambos casos la aproximación ha consistido en revisar conceptos básicos, tales como en el primero el de adaptación (R.L Day et al.2003) y en el segundo algunos supuestos como el llamado «egoísmo» tanto en la biología como en la economía. El texto que sigue busca presentar y valorar el planteamiento de una teoría naturalista de la evolución que debe tanto a la teoría tradicional de la evolución por selección natural, como a la ascendente teoría de la construcción de nicho que propone este proceso como principio explicativo complementario de la selección natural en la evolución. Más que una especialidad biológica dentro del marco evolucionista la construcción de nicho es una perspectiva de pensamiento que debe orientar la ejecución en concreto de las investigaciones. ${ }^{1}$

1 «La teoría de construcción de nicho es una rama emergente de la biología evolucionista que pone el énfasis en la capacidad de los organismos de modificar la selección natural en su ambiente y por esa razón actúan como co-directores de su propia evolución y la de otras especies. Ha de ser considerada más como una forma alternativa de pensar acerca de problemas 


\section{SELECCIÓN NATURAL Y CONSTRUCCIÓN DE NICHO}

En el año 2003 Odling-Smee, Laland y Feldman publicaron su conocido libro Niche Construction. The neglected process in evolution, en cuya introducción afirman que los organismos juegan dos papeles en la evolución:

[...] el primero consiste en portar los genes; los organismos sobreviven y se reproducen según el azar y las presiones selectivas naturales en sus ambientes [...]. El segundo papel de los fenotipos en la evolución no está bien descrito ni bien entendido por los biólogos evolucionistas y no ha sido objeto de mucha investigación. Lo llamamos «construcción de nicho» (F.J. Odling-Smee et al. 2003, p. 1).

La idea de la construcción de nicho constituye el polo opuesto, en las interacciones entre los organismos y sus medios, de la selección natural entendida como la reproducción diferencial entre variedades de las diferentes especies en virtud de las ventajas que ciertas características de los organismos favorecen (o, en caso contrario, perjudican) la supervivencia de unas u otras. En la selección natural, el medio o ambiente como conjunto o sistema de restricciones juega el papel fundamental y se considera que cualesquiera transformaciones del mismo por parte de los organismos no son suficientemente importantes como reacción y contrapeso a esas restricciones. La tesis de la construcción de nicho es justamente que esas transformaciones son relevantes en el proceso general de la evolución porque contribuyen incluyendo en el mismo la transformación de los ambientes como resultado de la acción de los organismos. Por tanto, la idea de la construcción de nicho no niega la acción de la selección natural, sino que la complementa de forma recíproca. ${ }^{2}$

Esa acción transformadora del medio hace que el organismo deje de ser un mero vehículo (Dawkins) y sea un verdadero interactor (Hull), de forma tal que si el medio alcanza los genes a través de las fronteras orgánicas, tampoco los organismos están encapsulados en sus fronteras aparentes, sino volcados a través de ellas en sus efectos en los medios correspondientes. Esta proyección causal no ha sido negada por los evolucionistas más seleccionistas. Prueba de ello es el bien conocido libro de Dawkins The Extended Phenotype (1982). La cuestión no es tanto el hecho de la eficacia del organismo sobre el medio, de que trata precisamente ese libro, sino la interpretación en términos de la eficacia biológica de los genes de esos organismos que trascienden sus fronteras. Es decir,

de la evolución que como un campo separado de la investigación evolucionista» (K.N. Laland y M.J. O’Brian 2010, p. 303).

2 K. Sterelny (2005, p. 21) expresa esta relación en el título de su artículo como la de la mutua producción de organismos y ambiente: «Made by Each Other: Organisms and their Environment». 
que la influencia de las transformaciones del medio se canaliza en el curso de la herencia genética y no constituye por sí misma otra forma de herencia. ${ }^{3}$ Por el contrario, la tesis de la construcción de nicho abona la afirmación de un segundo tipo de herencia no genética, sino ambiental o ecológica. Los organismos no sólo heredan los genes, sino también los ambientes. En resumen, dos procesos de trasmisión en una interacción que, si no fuera porque la palabra ha sido tan manoseada que a veces se torna trivial, podría considerarse dialéctica. Me acojo aquí a la legitimidad de usar el vocablo basándome en la verosimilitud que le dan nombres como Lewontin, el reconocido precursor de la tesis de la construcción de nicho ${ }^{4}$, y en España Cordón $(1966,1982)^{5}$ con la tesis de la evolución conjunta de los animales y su medio. Con la cautela adicional de que esta dialéctica -una idea filosófica- tiene un valor regulativo y heurístico, y que sólo el desarrollo bajo esa regla de conceptos científicos verdaderamente operativos pueden añadir a su sentido filosófico un conjunto de verdades científicas. ${ }^{6}$

\section{LOS ORGANISMOS FUERA DE Sí}

La literatura reciente está plagada de expresiones como «fenotipo ampliado», «organismo ampliado» (Turner 2000), etc. Hay que reconocer, sin embargo, que las ideas tras estas expresiones no son tan recientes. En cuanto a la segunda expresión, literalmente tomada, encontramos un recuento de antecedentes en S. Rattasepp (2010), que incluye a William James, Ernst Mach, Arthur Bentley,

3 Una posición que R. Dawkins (2004) reitera y defiende, muchos años después, como un planteamiento «disciplinado» en un trabajo donde responde a varias versiones, entre ellas la de la construcción de nicho, ampliadas de la teoría de la evolución seleccionista. Allí Dawkins distingue entre alteración y construcción de nicho. Lo primero es universal aunque trivial y lo segundo lo considera perfectamente asumible dentro de su noción de fenotipo ampliado.

Aunque Dawkins sea el contrincante más conocido del gran público, es toda la tradición seleccionista la que no ve necesidad de incorporar la construcción de nicho a la teoría de la evolución. K.N. Laland y K. Sterelny publicaron en un estudio siete puntos básicos para descartar o no la construcción de nichos en la teoría de la evolución. No tengo espacio para entrar en el detalle, pero recomiendo su lectura en una revista tan poco sospechosa como Evolution (cf. K.N. Laland and K. Sterelny 2006).

4 La cita habitual es a R. Lewontin (1983), aunque la base del argumento y la ilustración de los sistemas de ecuaciones se encuentra también en R. Levins y R. Lewontin (2000, pp.104 y ss.)

5 Introduzco a Cordón por la justificación del empleo del término «dialéctica», aunque reconozco que su caso merece un estudio aparte.

6 Buen ejemplo de ello es lo sucedido con la llamada lógica dialéctica. La idea general de una lógica dialéctica no pasó de ser un aliciente para el desarrollo de lógicas que, como las llamadas lógicas paraconsistentes, admiten contradicciones, salvo las fuertes del sistema. Algo semejante es lo que sugiero aquí, aunque en el caso de las lógicas la noción de dialéctica sea recta y en este caso puede ser oblicua o analógica. 
John Dewey, Francis Sumner, Andras Angyal y A. Irving Hallowell. Lo que todos denuncian en sus planteamientos es la llamada concepción morfológica de organismo, para la cual la piel es la frontera que separa organismo y medio de forma tajante, creando un dentro y un fuera. ${ }^{7}$

Por otra parte, en otro lugar yo mismo (J.R. Álvarez 1985, pp. 120 y ss.) me ocupé de las esferas orgánicas de Nicolai Hartmann y de los campos de comportamiento de François Meyer. El primero afirmaba: «el límite material del cuerpo no coincide con el límite del individuo viviente. El organismo está, mediante sus funciones vitales, en una relación con el mundo físico que va muy lejos» (N. Hartmann 1964, p.19). La figuralidad del cuerpo individual se corrige por la constitución de una esfera consistente en el entramado de acciones y reacciones que se extiende más allá de los límites corporales del individuo. El individuo traza a su alrededor una esfera vital que se caracteriza por su centralidad y su autotrascendencia. El individuo se constituye en centro de su esfera, creando una especie de interioridad de la misma, y complementariamente una nueva exterioridad, pero al lado de esta interioridad existe la trascendencia: «el complexo orgánico es el ente que en sus funciones esenciales internas existe fuera de sí» (ibid., 1964, p. 22).

El segundo sostenía que el organismo, al recurrir a su medio externo para obtener las sustancias necesarias, «desborda sus dimensiones anatómicas y fisiológicas para integrar una nueva dimensión, la del comportamiento» (F. Meyer 1967, p. 785). Así se logra distinguir un campo biológico que abarca tanto al organismo individual como a los elementos del medio que forman con él un sistema. «El sistema propiamente biológico se prolonga, por tanto, de forma paradójica más allá de los límites del organismo en sentido estricto. El campo de comportamiento se presenta como una red estructural proyectada sobre el medio por el ser vivo» (ibid.).

No obstante, puede señalarse que, en el pasado reciente que más nos afecta, propiamente hasta el libro de Dawkins (1982) no se ofrece una exposición en términos de conceptos biológicos actuales de las unidades orgánicas ampliadas o extendidas. ${ }^{8}$

7 «Y todos los autores que tratan el tema del «organismo ampliado» están insatisfechos con el principio general de que el organismo está separado de su ambiente por una frontera o barrera física concreta entre ambos, de forma que el organismo estaría «dentro» y el ambiente «fuera»-que uno sería precisamente lo que el otro no es. En lo que sigue todos los investigadores se caracterizan por su abandono de la llamada «concepción morfológica» de los organismos (Palmer 2004:321), es decir, la idea de que, con vistas a describir globalmente un organismo como unidad de investigación, basta con señalar un elemento particular de su anatomía -en este caso, su piel» (S. Rattassep 2010, p. 34).

8 La catarata posterior de literatura sobre las unidades extendidas o individuos rectificados -con especial relieve, las referentes a la extended mind desde el artículo seminal de A. 
La idea principal que dirige la teoría de la construcción de nicho es la de la proyección causal que extiende la eficacia operativa de las unidades orgánicas y la transformación de los medios. El componente proyectivo entendido como extensión hace de las unidades orgánicas no individuos cerrados, sino focos operativos cuyas fronteras con los medios son difusas. El efecto transformador hace de la relación entre organismos y medios una relación técnica en que los organismos alteran el ambiente de forma que, para sus descendientes y para otros miembros de especies que cohabitan en el ambiente, constituyen una herencia ambiental o ecológica. Si la selección natural presenta el ambiente como dominante y filtro a través de los fenotipos de los genes correspondientes, la construcción de nicho considera a los organismos como una especie de ingenieros ambientales que dejan como legado ambientes diferentes.

\section{LA APROXIMACIÓN ENTRE LAS CIENCIAS BIOLÓGICAS Y CIENCIAS HUMANAS}

\section{IV.1. LAS RELACIONES RESTRICTIVAS Y TÉCNICAS EN LA INTERSECCIÓN DE LOS PRINCIPIOS DE LAS CIENCIAS NATURALES Y LAS CIENCIAS HUMANAS}

En diversos lugares (especialmente, $c f$. J.R. Álvarez 1988) y repetidas ocasiones he recurrido a una clasificación de las ciencias que se basa en la autocomposición del conjunto de términos en que se establecen las tres conocidas relaciones del proyecto semiótico de C.W. Morris, a saber, semántica, pragmática y sintáctica. El conjunto potencia de \{signos, objetos, sujetos\} es el de las nueve relaciones binarias que figuran en la tabla 1 :

\begin{tabular}{|l|l|l|l|}
\hline & SIGNOS & OBJETOS & SUJETOS \\
\hline SIGNOS & sintáctica & representativa & normativa \\
\hline OBJETOS & incorporativa & objetiva & restrictiva \\
\hline SUJETOS & simbólica & técnica & social \\
\hline
\end{tabular}

Tabla 1

Las nueve relaciones de la tabla se caracterizan, en una primera aproximación, por su agrupación en tres subconjuntos de cinco relaciones, atendiendo a que contengan uno de los tres elementos del conjunto inicial aquí desarrollado. Es decir hay relaciones que contienen signos, que contienen objetos y que contienen sujetos. Esto permite establecer un paralelo con los principios de las

Clark y D. Chalmers (1998) y en la síntesis ampliada de la teoría de la evolución (M. Pigliucci y G. B. Müller 2010)- ocuparían un espacio aquí no disponible. 
teorías de las distintas ciencias, que conduce, respectivamente, a tres clases de ciencias: las ciencias naturales, semióticas y humanas.

Las ciencias cuyos principios teóricos pueden asociarse a las relaciones que contienen objetos: representativas, incorporativas, objetivas, restrictivas y técnicas, constituyen el conjunto de las ciencias naturales. Las ciencias cuyos principios teóricos pueden asociarse a las relaciones que contienen signos: sintácticas, representativas, normativas, incorporativas y simbólicas, constituyen el conjunto de las ciencias semióticas. Las ciencias cuyos principios teóricos pueden asociarse a las relaciones que contienen sujetos: normativas, restrictivas, simbólicas, técnicas y sociales, constituyen el conjunto de las ciencias humanas.

Las ciencias naturales y las ciencias semióticas comparten principios representativos e incorporativos: éste es el punto de vista semántico. Las ciencias semióticas y las humanas comparten principios normativos y simbólicos: éste es el punto de vista pragmático. Las ciencias naturales y las ciencias humanas comparten principios restrictivos y técnicos: en ausencia de nombre procedente de la Semiótica de Morris, se le denomina punto de vista económico, en virtud de que los principios restrictivos (limitaciones del entorno a las acciones) y los técnicos (acciones de transformación del entorno) componen, en el sentido etimológico de «oikos», la idea de que los elementos del entorno no sólo son limitaciones, sino también disponibilidades (recursos), entre las cuales se ha ido configurando nuestra casa, nuestro mundo, cada vez más artificial (por la tecnología) a partir de una naturaleza que hay concebir en el límite de un regreso reflexivo, cuando se quiere hablar de la naturaleza o, por contraste, siempre relativamente a técnicas determinadas para las cuales una naturaleza es a la vez un conjunto de limitaciones y disponibilidades En la mutua remisión de las relaciones inversas restrictivas y técnicas cabe la posibilidad de alcanzar un entendimiento de la oposición selección natural/construcción de nicho.

\section{IV.2 . LA DIALÉCTICA ECOLÓGICO-TÉCNICA}

Entre los castores que construyen presas en los ríos y los miembros de la especie homo sapiens que construyen viaductos y polígonos industriales media una gran distancia que no ha impedido, sin embargo, la proyección analógica de la transformación del mundo por el trabajo en la transformación del medio a través de la actividad de los organismos: por ejemplo el tratamiento digestivo del suelo de las lombrices de tierra ya estudiadas por Darwin. Entre selección natural y construcción de nicho se establece la dialéctica de la evolución, un proceso de realimentación no trivial que tiene un intercambio peculiar entre la naturalización de la acción y la operativización de la naturaleza. Es bien conocida la utilización por parte de Darwin de la selección artificial como modelo de la selección natural y la extensión a todas las poblaciones orgánicas de la lucha 
por la existencia maltusiana concebida para las poblaciones humanas $(C f$. J.R. Álvarez 2009, 2010). En la teoría de la construcción de nicho, los constructores de referencia no son ni los castores, ni los macacos, ni las lombrices de tierra, sino los homines sapientes. Hay aquí una naturalización de la técnica humana que se consigue con la simultánea operativización de las actividades orgánicas de especies no humanas en sus medios respectivos. Por eso no debe de extrañar que se plantee la extensión de la construcción de nicho a las ciencias humanas como la arqueología ( $C f$. K.N. Laland y M.J. O’Brian 2010), porque precisamente es devolver la idea generalizada a sus orígenes particulares una vez que éstos aparecen ahora como casos a los que se aplica la idea general, al modo como se aplica a las poblaciones humanas la idea general de la lucha por la existencia desde las que fue extendida por Darwin a todos los demás (Cf. J.R. Álvarez 2009).

Sin duda se trata de un procedimiento analógico redoblado de una naturalización por absorción en un género de referencia de la especie a la que conviene literalmente la cualidad o característica en cuestión. Ejemplificada curiosamente en un contexto semejante encontramos esta idea en la Crítica del Juicio (I. Kant $1790, \S 90$, nota primera):

Analogía (en significación cualitativa) es la identidad de la relación entre fundamentos y consecuencias (causas y efectos) en cuanto tiene lugar prescindiendo de la diferencia específica de las cosas o de aquellas cualidades en sí que contienen el fundamento de consecuencias semejantes (es decir, consideradas fuera de esa relación). De ese modo, para las acciones artísticas (Kunsthandlungen) de los animales, comparadas con las de los hombres, pensamos el fundamento desconocido para nosotros de esos efectos, en los primeros, como un análogo de la razón, apoyándonos para ello en el fundamento conocido de los efectos semejantes en los hombres, y queremos, al mismo tiempo, indicar con eso que el fundamento de la facultad artística (Kunstvermögen) de los animales, con la denominación de instinto es, en realidad, específicamente distinto de la razón, aunque tiene una relación semejante con el efecto (comparando el edificio que levanta el castor con el que levanta el hombre). De eso, de que el hombre para su edificio necesita razón, no puedo concluir que el castor tenga que tener también razón, y llamar eso una conclusión por analogía. Pero del modo semejante de obrar de los animales (cuyo fundamento no podemos percibir inmediatamente) comparado con el de los hombres (de que tenemos inmediata conciencia), podemos con toda corrección, concluir por analogía, que los animales también actúan según representaciones (no son, como quiere Descartes, máquinas), y prescindiendo de su diferencia específica, son, sin embargo, idénticos a los hombres, según el género (como seres vivos). ${ }^{9}$

9 Cito según la traducción de M. García Morente, con alguna modificación. 
Donde dice «arte» bien podría decir «técnica», pues en ambos casos se trata de una acción de transformación del entorno según representaciones. Analogía no es identidad, pero lo que es analógico entre especies es idéntico en el género de los seres vivos que aquí el pensador de Könisberg caracteriza, tal vez con excesiva amplitud, como agentes representativos. La analogía kantiana es representacional, la de los teóricos de la construcción de nichos es transformacional: las lombrices se «trabajan» el terreno. Organismo y agente transformador del medio coinciden, son genéricamente idénticos y específicamente análogos.

Basada en la analogía, apoyada en los principios ecológicos y técnicos, la dialéctica entre selección natural y construcción de nicho requiere, como se indicó en la introducción, una reformulación del concepto de adaptación que no se limite a la noción de una causalidad unidireccional en que los ambientes filtran los genes de ascendientes a descendientes. Como se expresan Laland y Sterelny:

La perspectiva convencional explica esta complementariedad <organismo-medio> como algo que resulta únicamente de una selección natural que esculpe los organismos a partir de los ambientes. Inversamente, la perspectiva de la construcción de nicho reconoce dos rutas lógicamente diferentes hacia un ajuste organismoambiente: la selección natural (que impulsa la acomodación asimétrica al ambiente) y la construcción de nicho. La última implica la construcción, disminución, modificación o regulación de los recursos por parte de los organismos en sus ambientes, de forma que mejora su eficacia biológica (fitness) en el ambiente. (K.N. Laland y K. Sterelny 2006, p.1758).

¿Cuál es el complemento de la acomodación que conduce a mejorar la eficacia biológica? Este es el último extremo que debe sustanciarse antes de concluir.

\section{LA ADAPTACIÓN REVISADA}

El principio explicativo de la selección natural que Darwin introdujo en el bloque causal de El origen de las especies (capítulos I-IV, $c f$. J.R. Álvarez 2010) es un principio ecológico; el principio de la construcción de nicho es un principio técnico que para el darwinismo de más estricta observancia queda incorporado como mucho al planteamiento de Dawkins de fenotipos ampliados, y en esa incorporación queda neutralizado. Para J. Moreno (2008), que enfoca la evolución desde la ecología, la construcción de nichos en un tiempo $t$ es el resultado de la acción de los organismos seleccionados en un tiempo $t-1$, es 
decir, no es otra cosa que «el producto de la selección natural previa» (ibid., 2008, p. 29).

Muy al contrario, para los defensores de la teoría de construcción de nicho la teoría de la evolución reducida a la unicidad del principio explicativo de la selección natural es una teoría incompleta que ha de corregirse con la introducción en parangón del principio operacional de la transformación del medio por los organismos y el establecimiento de la relación dialéctica o interactiva entre herencia genética y herencia ambiental. Son conscientes de que su planteamiento no ha de convencer solamente con palabras, sino con hechos: por medio del desarrollo de los modelos adecuados que haga no solamente de su alegato un discurso retórico, sino una ejecución científica con resultados tan solventes como los que pueda producir la aplicación operativa del principio de selección natural. Es más, sin la introducción efectiva de principio de construcción de nicho tampoco la teoría de la evolución podrá alcanzar sus fines epistemológicos.

Con los principios conjugados de selección natural y construcción de nicho es necesario revisar el concepto de adaptación, que con la sola referencia a la causalidad de la selección quedaba reducida a la influencia del medio sobre los organismos. La construcción de nichos obliga a reconocer el carácter dual de la adaptación. De esto también existen antecedentes, como de casi todo. El gran psicólogo evolutivo del siglo pasado, Jean Piaget, que empezó siendo malacólogo, comprendió la adaptación como la síntesis de dos procesos opuestos, a saber, la acomodación del organismo al medio y la asimilación del medio por el organismo ( $C f$ J. Piaget 1969). La semejanza entre acomodación y selección natural, y entre asimilación y construcción de nicho, no es un paralelo perfecto, pero no es posible pasarla por alto (Cf. S.T. Parker 2005).

En efecto, la acomodación del organismo al medio es claramente asociable a un principio restrictivo en el sentido en que este tipo de principios se entendió en la sección IV.1 y a la selección natural (tanto en su sentido negativo de filtro como positivo de canalización). La asimilación del medio por el organismo, en cambio, lo es a un principio técnico según el cual el medio o ambiente es también una fuente de recursos transformables y al principio construcción de nicho. La interacción no trivial entre selección natural y construcción de nicho con las consecuentes herencias genética y ambiental constituye una dinámica interna a un proceso de adaptación que ya no se reduce a la acomodación.

\section{CONCLUSIÓN}

La adaptación así entendida integra una interacción recurrente entre selección natural y construcción de nicho. Este sería el sentido «dialéctico» a que apuntaban los planteamientos iniciales de Lewontin en los años ochenta del siglo pasado, aunque como es conocido existen problemas categoriales de 
aplicación del concepto de dialéctica a la naturaleza (el consabido problema de una dialéctica de la naturaleza). ${ }^{10}$

Además, la teoría de construcción de nicho, como ya se expuso, implica un proceso analógico en el cual lo que empieza siendo ejemplo (las acciones técnicas de los animales, eminentemente las de los humanos) termina por ser recuperado como un caso particular del concepto general de construcción de nicho. Entre el origen (el ejemplo) y el término final (el concepto) se produce la absorción del primero por el segundo. ${ }^{11}$ La dialéctica entre ejemplos y conceptos, como se señaló, existe también en la relación entre la selección artificial y la selección natural darwinianas. En este contexto el concepto de dialéctica no plantea problemas categoriales de aplicación y el ejemplo se transforma en caso a través del concepto por un procedimiento analógico.

Para finalizar, la teoría de la evolución por selección natural y construcción de nicho, basada en principios ecológicos y técnicos, proporciona la base de un panorama unificador de las ciencias biológicas y las ciencias humanas, precisamente porque se desarrolla sobre los principios que ambas comparten. Ésta es otra manera de volver a abrir la puerta al tratamiento paralelo de la evolución biológica y la evolución cultural, de la tortuga y la liebre de D.P. Barash (1987), tras intentos más bien fallidos como la memética y afines.

\section{REFERENCIAS BIBLIOGRÁFICAS}

ÁLVAREZ, J. R. (1985), «Fenomenologías, ontologías y metodologías biológicas», en Contextos, (1985), III/6, pp. 115-150. , (1988), Ensayos metodológicos, León: Universidad de León. ,(2009), «De aquel Darwin tan singular al darwinismo universal: la problemática naturalización de las ciencias humanas», en Ludus vitalis, XVII, (2009), 32, pp. 307-326.

,(2010), «La selección natural: lenguaje, método y filosofía», en Éndoxa, (2010), 24, pp. 91-122.

BARASH, D.P. (1987), La liebre y la tortuga. Cultura, biología y naturaleza humana, traducción de A. Ortuño y J. Vicuña, Barcelona: Salvat.

10 Aquí sería oportuno tratar en detalle los planteamientos de F. Cordón (op. cit,) que sí llevan esa dirección, pero que en este espacio no tienen cabida.

11 G. Bueno (1985) empleó el vocablo «absorción» en este sentido hace ya muchos años, contraponiendo los esquemas de reducción a los esquemas de absorción, entre los cuales estableció una relación dialéctica. 
BUENO, G. (1985), «La dialéctica de la reducción-absorción en la historia del pensamiento», prólogo a Javier de Lorenzo, El racionalismo y los problemas del método, Madrid: Cincel, pp. 9-14.

CLARK, A. and CHALMERS, D. (1998), «The extended mind », en Analysis (1998), 58,1, pp. 7-19.

CORDÓN, F. (1966), La evolución conjunta de los animales y su medio, Barcelona:

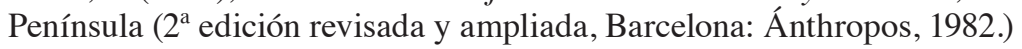

DAWKINS, R. (1982), The Extended Phenotype. The Gene as the Unit of Selection, Oxford: Oxford University Press. , (2004), «Extended Phenotype - But Not Too Extended. A Reply to Laland, Turner and Jablonka», Biology and Philosophy, (2004), 19, pp. 377-396.

DAY, R.L., LALAND, K.N., ODLING-SMEE, F. J. (2003) «Rethinking adaptation: the niche-construction perspective», en Perspectives in Biology and Medicine, 46, 1 (2003), pp.80-95

HARTMANN, N. (1964), Ontología. V. Filosofía de la naturaleza: categorías organológicas, traducción de J. Gaos, México D.F.: Fondo de Cultura Económica.

HARVEY, D. (1993), «The Nature of Environment: The Dialectics of Social and Environmental Change», Socialist Register, 29 (1993), pp. 1-51, accessible en http:// socialistregister.com/index.php/srv/issue/view/427. (Consultado 16/06/2012)

KANT, I. (1790), Kritik der Urtheiskraft, en Kants gesammelte Schriften, Band V, Berlín: Reimer, 1913, pp. 165-485 (Traducción de M. García Morente, Madrid: Espasa-Calpe, 1977).

LALAND, K.N. and STERELNY, K. (2006), «Perspective: Seven reasons (not) to neglect niche construction», en Evolution (2006), 60, 9, pp. 1751-1762.

LALAND, K,N. and M.J. O'BRIAN (2010): «Niche Construction Theory and Archaeology», Journal of Archaeological Methodological Theory, (2010), 17, pp. 303-322.

LEVINS, R. and LEWONTIN, R.(1985), The Dialectical Biologist, Cambridge (MA): Harvard University Press.

LEWONTIN, R.C. (1983). «Gene, organism, and environment», en Bendall, D.S. (ed.) Evolution from Molecules to Men, Cambridge: Cambridge University Press, pp. 273-285.

MEYER, F. (1967), «Situation épistémologique de 1a Biologie», en Piaget, J. (comp.), Logique et connaissance scientifique, Paris: Gallimard, 1967, pp. 781-821.

MORENO, J. (2008), Los retos actuales del darwinismo. ¿Una teoría en crisis?, Madrid: Síntesis.

MORRIS, C.W. (1972), «Fundamentos de la teoría de los signos», traducción de E. Torrego, en GRACIA, F. (Comp.), (1972) Presentación del lenguaje, Madrid: Taurus, pp. 54-65.

ODLING-SMEE, F.J., LALAND, K.N., FELDMAN, M.W. (2003), Niche Construction. The neglected process in evolution, Princeton: Princeton University Press.

PIAGET, J. (1969), Biología y conocimiento, traducción de F. Rodríguez Aramburu, Madrid: Siglo XXI. 
RATTASEPP, S. (2010): «The Idea of extended Organism in $20^{\text {th }}$ Century Thought», Hortus semioticus, (2010) 6, pp. 31-39. Accesible en: http://www.academia. edu/935963/The_Idea_of_Extended_Organism_in_20th_Century_Thought (Consultado 6/12/2012)

PARKER, S.T., (2005), «Piaget's Legacy in Cognitive Constructivism, Niche Construction, and Phenotype Development and Evolution», en Parker, S.T., et al. (2005), Biology \& Knowledge Revisited, From Neurogenesis to Psychogenesis, Londres: Erlbaum, pp. 1-31.

PIGLIUCCI, M. and MÜLLER, G.B. (2010), Evolution. The Extended Synthesis, Cambridge (MA): MIT Press.

STERELNY, K. (2005), «Made by Each Other: Organisms and their Environment», Biology and Philosophy, (2005), 20, pp. 21-36.

TURNER, J.S. (2000), The Extended Organism. The Physiology of Animal-Built Structures, Cambridge (MA): Harvard University Press.

Juan Ramón Álvarez Bautista es Catedrático de Lógica y Filosofía de la Ciencia en el Departamento de Psicología, Sociología y Filosofía de la Universidad de León.

Líneas de Investigación:

Filosofía de la ciencia, Filosofía de la Biología, Filosofía de las ciencias sociales, Metodología de la ciencia.

Publicaciones recientes:

«La selección natural: lenguaje, método y filosofía», Éndoxa, 24 (2010), pp. 91-122.

«Criaturas de la naturaleza y criadores de cultura: de vuelta con la naturaleza humana», Scripta Philosophiae Naturalis, 1 (2012), pp. 13-27.

Dirección electrónica: juan-ramón.alvarez@unileon.es 